QUARTERLY OF APPLIED MATHEMATICS

VOLUME LXVII, NUMBER 3

SEPTEMBER 2009, PAGES 419-440

S 0033-569X(09)01129-1

Article electronically published on May 5, 2009

\title{
MODELLING SUPPLY NETWORKS WITH PARTIAL DIFFERENTIAL EQUATIONS
}

\author{
BY \\ C. D'APICE (Department of Information Engineering and Applied Mathematics, University of \\ Salerno, Fisciano (SA), Italy), \\ R. MANZO (Department of Information Engineering and Applied Mathematics, University of \\ Salerno, Fisciano (SA), Italy), \\ AND \\ B. PICCOLI (Istituto per le Applicazioni del Calcolo "Mauro Picone", Consiglio Nazionale delle \\ Ricerche, Roma, Italy)
}

\begin{abstract}
A continuum-discrete model for supply networks is introduced. The model consists of a system of conservation laws: a conservation law for the goods density and an evolution equation for the processing rate. The network is formed by subchains and nodes at which, motivated by real cases, two routing algorithms are considered: the first maximizes fluxes taking into account the goods' final destinations, while the second maximizes fluxes without constraints. We analyze waves produced at nodes and equilibria for both algorithms, relating the latter to production rates in real supply networks. In particular, we show how the model can reproduce the well-known Bullwhip effect.
\end{abstract}

1. Introduction. A supply network can be considered as an organization of activities that performs the functions of materials procurement, their transformation into intermediate and finished goods, and the distribution of these finished products to customers. It is evident that the term supply network can be seen in a very general way, since it is about the goods' production and their distribution to the final user.

In recent years, the interest of the scientific community for supply chains and networks modelling has become greater and greater. The main aim is to plan supply networks in such way as to reduce the dead times and to avoid bottlenecks, obtaining as a result a better coordination leading to the optimization of the production process of a given good. Supply networks modelling is characterized by different mathematical approaches: on the one hand, there are discrete event simulations based on considerations of individual

Received October 3, 2007.

2000 Mathematics Subject Classification. Primary 35L65, 90B30.

Key words and phrases. Conservation laws, supply networks.

E-mail address: dapice@diima.unisa.it

E-mail address: manzo@diima.unisa.it

E-mail address: b.piccoli@iac.cnr.it 
parts; on the other hand, continuous models (for a general overview, see [1, 2], 3], 4]), based on partial differential equations, have been introduced. Probably the first paper for supply chains in this direction was [2, where the authors, taking the limit on the number of parts and suppliers, have obtained a conservation law, whose flux is described by the minimum among the parts density and the maximal productive capacity.

Due to the difficulty of finding a solution for the general equation proposed in [2], other fluid dynamic models for supply chains were introduced in [6, [9] and [13. The work 9] is based on a mixed continuum-discrete model; i.e., the supply chain is described by continuous arcs and discrete nodes. This means that the load dynamics is solved in a continuous way on the arcs, and at the nodes imposing the conservation of the goods' density, but not of the processing rate. In fact, each arch is modelled by a system of two equations: a conservation law for the goods' density, and an evolution equation for the productive capacity. Possible choices of solutions at nodes guaranteeing the conservation of fluxes are discussed, and a Riemann Solver is defined, fixing the rule:

SC1. The incoming density flux is equal to the outgoing density flux. Then, if a solution with only waves in the density exists, then such a solution is taken; otherwise the minimal processing rate wave is produced.

Moreover, the existence of solutions to Cauchy problems was proved. The paper 13 . considers a conservation law, with constant processing rate, inside each supply subchain, with an entering queue for exceeding parts. The dynamics at a node is solved considering an ODE for the queue. Some optimization techniques for the model described in 13 . are developed in [14, while the existence of solutions to Cauchy problems with the front tracking method is proved in [15. It is evident that the models described in [9] and [13] complete each other. In fact, the approach of [13] is more suitable when the presence of a queue with buffer is fundamental to manage goods' production. The model of $\underline{9}$, on the other hand, is useful when there is the possibility of reorganizing the supply chain: particularly, the productive capacity can be readapted for some contingent necessity.

Starting from the model introduced in [9] and fixing the rule that the objects are processed in order to maximize the flux, two different Riemann Solvers are described and equilibria at a node are discussed in [6]. Moreover, discretization algorithms to find an approximate solution to the problem are described, and numerical experiments on sample supply chains are reported and discussed for both the Riemann Solvers.

While the papers [2, 6], 9] treat the case of chains, i.e. sequential processors, modelled by a real line seen as a sequence of subchains corresponding to real intervals, the model in 13 and the extended results in [14, 15] refer to networks.

The aim of this paper is to extend the continuum-discrete model of [6] and [9], regarding sequential supply chains, to supply networks which consist of subchains and two types of nodes: nodes with one incoming subchain and more outgoing ones and nodes with more incoming subchains and one outgoing subchain. In fact, these two types of nodes are the most common in real supply networks.

Definition 1.1 (Network definition). A supply network is a finite, connected directed graph consisting of a finite set of $\operatorname{arcs}$ (subchains) $\mathcal{I}=\left\{I_{k}: k=1, \ldots, N+1\right\}$ and a finite set of junctions $\mathcal{P}$. 
On each subchain the load dynamics is given by a continuum system of type

$$
\begin{aligned}
& \rho_{t}+f_{\varepsilon}^{k}(\rho, \mu)_{x}=0, \\
& \mu_{t}-\mu_{x}=0,
\end{aligned}
$$

where $\rho$ is the density of objects processed by the supply chain network, $\mu$ is the processing rate and $f_{\varepsilon}^{k}$ is the flux given by

$$
f_{\varepsilon}^{k}(\rho, \mu)= \begin{cases}\rho, & 0 \leq \rho \leq \mu \\ \mu+\varepsilon(\rho-\mu), & \mu \leq \rho \leq \rho_{k}^{\max }\end{cases}
$$

with $\rho_{k}^{\max }$ and $\mu_{k}^{\max }$ the maximum density and processing rate on the subchain $I_{k}$.

We interpret the evolution at a node $P$, thinking of it as a Riemann Problem (RP) for the density equation (1.1) with $\mu$ data as parameters. The Riemann Problems are solved fixing two "routing" algorithms:

RA1. Goods from an incoming subchain are sent to outgoing ones according to their final destination in order to maximize the flux over incoming subchains. Goods are processed in order of arrival time (FIFO policy).

RA2. Goods are processed by arrival time (FIFO policy) and are sent to outgoing subchains in order to maximize the flux over incoming and outgoing subchains.

The two algorithms were already used in [10] for the analysis of packet flows in telecommunication networks. Notice that the second algorithm allows the redirection of goods, taking into account possible high loads of outgoing subchains. For both routing algorithms the flux of goods is maximized considering one of the two additional rules, SC2 and SC3 (see [9]):

$\mathrm{SC} 2$. The objects are processed in order to maximize the flux with the minimal value of the processing rate.

SC3. The objects are processed in order to maximize the flux. If a solution with only waves in the density $\rho$ exists, then such a solution is taken; otherwise the minimal $\mu$ wave is produced.

The rules SC2 and SC3 seem to be more elastic than SC1, allowing more rich dynamics. According to these routing algorithms we define Riemann Solvers and discuss the waves formation at nodes. The detailed analysis of waves permits us to better visualize and understand the dynamics effects on subchains of the defined Riemann Solvers. We provide explicit examples to illustrate the differences between RA1 and RA2 and also between $\mathrm{SC} 2$ and $\mathrm{SC} 3$.

Then we consider generic equilibria with active and not active constraints for the maximization problem solved at nodes. We relate the found results with the counterpart in real supply networks. Then we show that SC3 reproduces the well-known Bullwhip effect (see [8, 11, 12, 17, 18, 20]); i.e., under certain conditions (delays in adaptation of production or delivery rates), the oscillations in delivery and in the resulting inventories (stock level of the products) grow from one producer to the next upstream one, leading to instability with respect to perturbation in the production rate. The latter confirms that SC3 appears to be the more realistic modelling choice.

The outline of the paper is the following. In Section 2 some examples of real supply networks are introduced in order to motivate the rules introduced to solve the dynamics 
at nodes. Section 3 gives the basic definitions of supply network, and Riemann Solvers. In Section 4. Riemann Solvers at junctions are defined for both algorithms, the wave formation is discussed and some numerical results are reported. Finally in Section 5 equilibria with active and not active constraints for the maximization problem are discussed and the Bullwhip effect is analyzed in the case of nodes with more incoming subchains and one outgoing subchain.

2. Real supply networks. In what follows, we want to give some examples of real supply networks and focus on some characteristics that can be useful for a mathematical description of production processes. In particular, the aim is to describe rules according to which the goods are addressed from incoming subchains to the outgoing ones in order to motivate the introduction of the two algorithms described in Section 1 .

Let us analyze a supply network for assembling wine bottles, described in Fig. 11(left). Bottles coming from arc $I_{1}$ are sterilized in node 1 . Then, the sterilized bottles, with
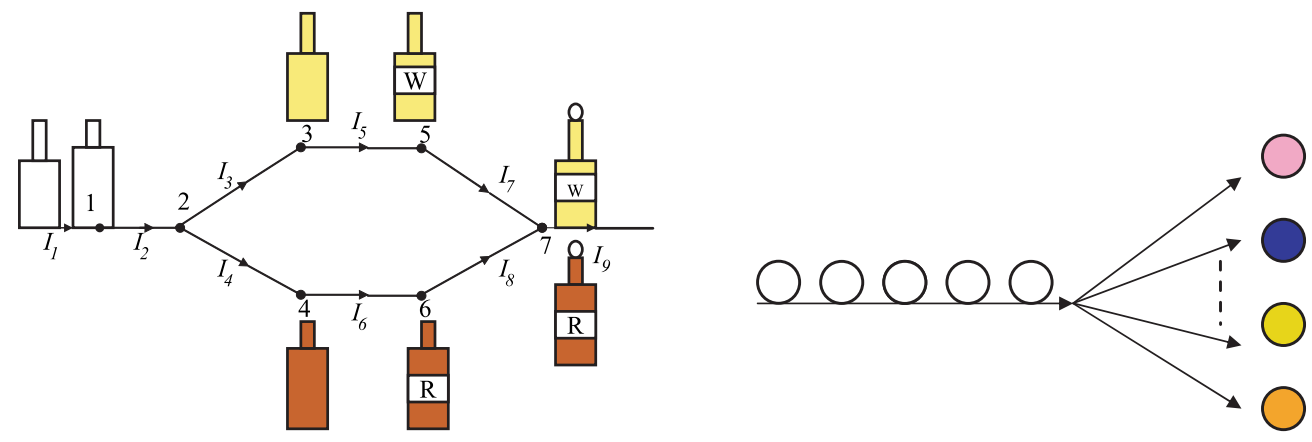

FIG. 1. Wine production network (left) and balls production network (right).

a certain probability $\alpha$ are directed to node 3, where white wine is bottled, and with probability $1-\alpha$ to node 4 , where the red wine is bottled. In nodes 5 and 6 , bottles are labelled, respectively, for white and red wine. Finally, in node 7 , produced bottles are corked. Assume that red and white wine bottles are produced using two different bottle shapes. The bottles are addressed from arc $I_{2}$ to the outgoing subchains $I_{3}$ and $I_{4}$ in which they are filled up with white or red wine according to the bottle shape and thus according to the final destination: production of white or red wine bottles. In a model able to describe this situation, the dynamics at node 2 is solved using the RA1 algorithm; in fact, the redirection of bottles in order to maximize the production on both incoming and outgoing subchains is not possible, since bottles with white and red wine have different shapes.

A supply network of beach balls production is considered in Fig. 1 (right). The white balls are addressed towards $n$ subchains in which they are colored using different colors. Since the aim of the factory is to maximize the balls production independently from the colors, a mechanism is realized which addresses the balls on the outgoing subchains by taking into account their loads in such way as to maximize flux on both incoming 
and outgoing subchains. It follows that a model realized to capture the behavior of the described supply network is based on rule RA2.

Let us now analyze an existing supply network where both algorithms show up naturally: the one for chips production of the San Carlo enterprise (see 21]). The productive process follows various steps, which can be summarized in this way: when potatoes arrive at the enterprise, they are subjected to a goodness test. After this test, everything is ready for chips production, which starts with potatoes being washed in drinking water. After washing potatoes, they are skinned off, rewashed and subjected to a qualification test. Then, they are cut in thin strips by an automatic machine, and, finally, washed and dried by an air blow. At this point, potatoes are ready to be fried in vegetable oil for some minutes and, after this, the surplus oil is dripped. Potatoes are then salted by a dispenser that nebulizes salt, spreading it on potatoes. An opportune chooser is useful to select the best products. The final phase of the process is given by potatoes confection. A simplified vision of the supply chain network is in Fig. 2 (top). In phases

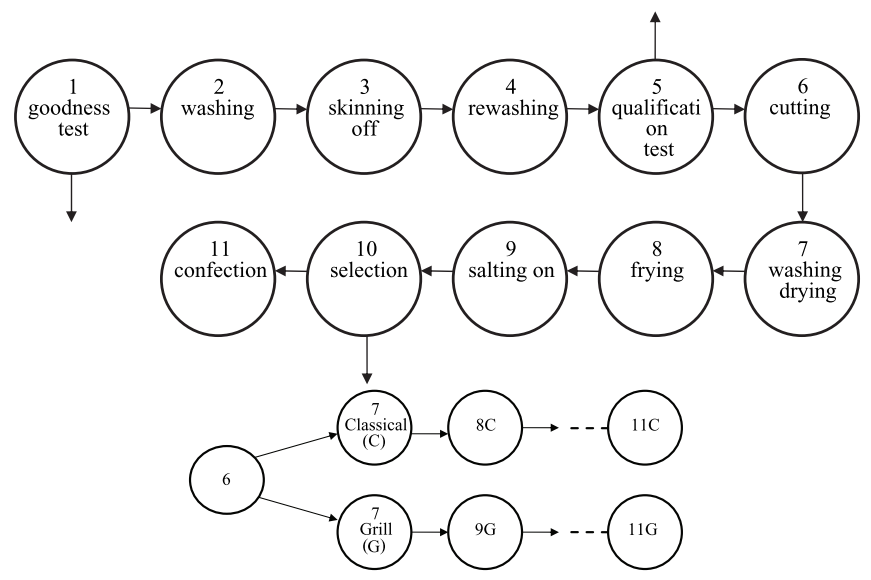

FIG. 2. Graph of the supply network for chips production (top) and possible subchains (bottom).

1,5 and 10, a discrimination is made in production in order to distinguish good and bad products. In such a sense, we can say that there is a statistical percentage $\alpha$ of the product that follows the production steps, while the percentage $1-\alpha$ is the product discarded (obviously, the percentage $\alpha$ can be different for different phases). Therefore, the goods routing in these nodes follows the algorithm RA1. On the other side, phase 6 concerns the potatoes cut: as the enterprise produces different types of fried potatoes (classical, grill, light, stick, etc.), different ways of cutting potatoes must be considered. Assume that, for simplicity, there are only two types of potato production. Then the supply network is as in Fig. 2 (bottom). If the aim is only the production maximization independently from the type, then the potatoes are addressed from node 6 towards the outgoing subchains according to the RA2 algorithm. 
3. Basic definitions. Let us consider a supply network consisting of $N+1$ subchains $I_{1}, \ldots, I_{N+1}$, modelled by real intervals $I_{k}=\left[a_{k}, b_{k}\right] \subset \mathbb{R}, k=1, \ldots, N+1, a_{k}<b_{k}$, possibly with either $a_{k}=-\infty$ or $b_{k}=+\infty$ and $M$ suppliers or processors $P_{1}, \ldots, P_{M}$ with certain throughput times and capacity. Each supplier processes a certain good, measured in units of parts. We assume that a node $P$ consists of a processor, which decides how to manage the flow among subchains, with a maximal processing rate $\mu$.

On each subchain $I_{k}$ we consider the system

$$
\left\{\begin{array}{l}
\rho_{t}+f_{\varepsilon}^{k}(\rho, \mu)_{x}=0 \\
\mu_{t}-\mu_{x}=0 .
\end{array}\right.
$$

Each subchain $I_{k}$ is thus characterized by a maximum density, a maximum rate and a flux $f_{\varepsilon}^{k}$. The flux is defined as in [9]; therefore:

(F) $f_{\varepsilon}^{k}(\rho, \mu)= \begin{cases}\rho, & 0 \leq \rho \leq \mu, \\ \mu+\varepsilon(\rho-\mu), & \mu \leq \rho \leq \rho_{k}^{\max },\end{cases}$ or alternatively

$$
f_{\varepsilon}^{k}(\rho, \mu)= \begin{cases}\varepsilon \rho+(1-\varepsilon) \mu, & 0 \leq \mu \leq \rho, \\ \rho, & \rho \leq \mu \leq \mu_{k}^{\max },\end{cases}
$$

where $\rho_{k}^{\max }$ and $\mu_{k}^{\max }$ are the maximum density and processing rate. From now on, we assume that $\varepsilon$ is fixed and, for simplicity, we drop the indices, thus indicating the flux by $f(\rho, \mu)$.

REMARK 3.1. It is possible to generalize all the following definitions and results to the case of different fluxes $f_{\varepsilon_{k}}^{k}$ for each subchain $I_{k}$ (also choosing $\varepsilon$ dependent on $k$ ). In fact, all statements are in terms of values of fluxes at endpoints of the subchains; thus it is sufficient that the ranges of the fluxes intersect. Moreover, we can consider different slopes $m_{k}$ for each subchain $I_{k}$, considering the following flux:

$$
f_{\varepsilon}^{k}(\rho, \mu)= \begin{cases}m_{k} \rho, & 0 \leq \rho \leq \mu, \\ m_{k} \mu+\varepsilon(\rho-\mu), & \mu \leq \rho \leq \rho_{k}^{\max }\end{cases}
$$

where $m_{k} \geq 0$ represents the velocity of each processor and is given by

$$
m_{k}=\frac{L_{k}}{T_{k}}
$$

with $L_{k}$ and $T_{k}$, respectively, the fixed length and processing time of the processor $P_{k}$.

We assume that the subchains are connected by some junctions. Each junction $J$ is given by a finite number of incoming subchains and a finite number of outgoing subchains; thus we identify $J$ with $\left(\left(i_{1}, \ldots, i_{n}\right),\left(j_{1}, \ldots, j_{m}\right)\right)$ where the first $n$-tuple indicates the set of incoming subchains and the second $m$-tuple indicates the set of outgoing subchains. Each subchain can be an incoming subchain at most for one junction and outgoing at most for one junction. Hence the complete model is given by a couple $(\mathcal{I}, \mathcal{P})$, where $\mathcal{I}=$ $\left\{I_{k}: k=1, \ldots, N+1\right\}$ is the collection of subchains and $\mathcal{P}$ is the collection of junctions.

The supply network evolution is described by a finite set of functions $\rho_{k}, \mu_{k}$ defined on $\left[0,+\infty\left[\times I_{k}\right.\right.$. On each subchain $I_{k}$, we say that $U_{k}:=\left(\rho_{k}, \mu_{k}\right):\left[0,+\infty\left[\times I_{k} \mapsto \mathbb{R}\right.\right.$ is a weak solution to (3.1) if, for every $C^{\infty}$-function $\varphi:\left[0,+\infty\left[\times I_{k} \mapsto \mathbb{R}^{2}\right.\right.$ with compact 
support in $] 0,+\infty[\times] a_{k}, b_{k}[$,

$$
\int_{0}^{+\infty} \int_{a_{k}}^{b_{k}}\left(U_{k} \cdot \frac{\partial \varphi}{\partial t}+f\left(U_{k}\right) \cdot \frac{\partial \varphi}{\partial x}\right) d x d t=0
$$

where

$$
f\left(U_{k}\right)=\left(\begin{array}{c}
f\left(\rho_{k}, \mu_{k}\right) \\
-\mu_{k}
\end{array}\right)
$$

is the flux function of the system (3.1). For the definition of an entropic solution, we refer to $[5]$.

A Riemann Problem for a scalar conservation law is a Cauchy problem for an initial data of Heaviside type, that is, piecewise constant with only one discontinuity. The solutions are formed by rarefactions and shocks (see [5, [7]).

Analogously, we call the Riemann Problem for a junction the Cauchy problem corresponding to an initial data which is constant on each supply subchain.

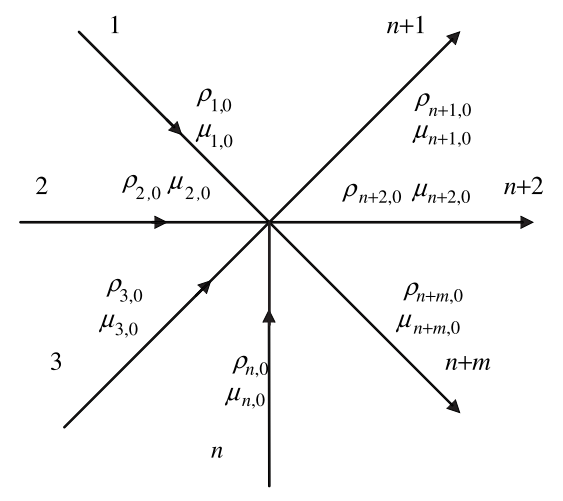

Fig. 3. A junction.

Definition 3.2. A Riemann Solver (RS) for the junction $P$ (see Fig. 3) with $n$ incoming subchains and $m$ outgoing ones consists of a map that associates to a Riemann datum $\left(\rho_{0}, \mu_{0}\right)=\left(\rho_{1,0}, \mu_{1,0}, \ldots, \rho_{n, 0}, \mu_{n, 0}, \rho_{n+1,0}, \mu_{n+1,0}, \ldots, \rho_{n+m, 0}, \mu_{n+m, 0}\right)$ at $P$ a vector $\left(\hat{\rho}_{0}, \hat{\mu}_{0}\right)=\left(\hat{\rho}_{1}, \hat{\mu}_{1}, \ldots, \hat{\rho}_{n}, \hat{\mu}_{n}, \hat{\rho}_{n+1}, \hat{\mu}_{n+1}, \ldots, \hat{\rho}_{n+m}, \hat{\mu}_{n+m}\right)$ so that the solution is given by the waves $\left(\rho_{i, 0}, \hat{\rho}_{i}\right)$ and $\left(\mu_{i, 0}, \hat{\mu}_{i}\right)$ on the subchain $I_{i}, i=1, \ldots, n$ and by the waves $\left(\hat{\rho}_{j}, \rho_{j, 0}\right)$ on the subchain $I_{j}, j=n+1, \ldots, n+m$. We require the consistency condition

(CC) $R S\left(R S\left(\left(\rho_{0}, \mu_{0}\right)\right)\right)=R S\left(\left(\rho_{0}, \mu_{0}\right)\right)$.

Riemann Solvers, according to algorithms RA1 and RA2, will be defined in the next section.

Once a Riemann Solver is assigned we can define the admissible solutions at $P$.

Definition 3.3. Assume a Riemann Solver RS is assigned for the supplier $P$. Let $U=\left(U_{1}, \ldots, U_{n+m}\right)$ be such that $U$ is of bounded variation for every $t \geq 0$. Then $U$ is an admissible weak solution of (3.1) related to RS at the junction $P$ if and only if the following property holds for almost every $t$. Setting

$$
\widetilde{U}_{P}(t)=\left(U_{1}\left(\cdot, b_{1}-\right), \ldots, U_{n}\left(\cdot, b_{n}-\right), U_{n+1}\left(\cdot, a_{n+1}+\right), \ldots, U_{n+m}\left(\cdot, a_{n+m}+\right)\right)
$$

we have $R S\left(\widetilde{U}_{P}(t)\right)=\widetilde{U}_{P}(t)$. 
Our aim is to solve the Cauchy problem on $[0,+\infty$ [ for a given initial and boundary data as in the next definition.

Definition 3.4. Given $\bar{U}_{k}: I_{k} \mapsto[0,1], k=1, \ldots, N+1$, measurable BV functions, a collection of functions $U=\left(U_{1}, \ldots, U_{N+1}\right)$, with $U_{k}:\left[0,+\infty\left[\times I_{k} \mapsto[0,1]\right.\right.$ continuous as a function from $\left[0,+\infty\right.$ [ into $L_{l o c}^{1}$ and $U_{k}(t, \cdot)$ a BV function for almost every $t$, then $U_{k}$ is an admissible solution to the Cauchy problem on the supply chain if it is a weak entropic solution to (3.1) on $I_{k}, U_{k}(0, x)=\bar{U}_{k}(x)$ a.e., and, at each supplier $P_{k}, U$ is an admissible weak solution.

4. Riemann Solvers for suppliers. In this section we discuss Riemann Solvers, which conserve the flux at nodes. We consider two kinds of nodes:

- a node with more incoming subchains and one outgoing one;

- a node with one incoming subchain and more outgoing ones.

Let us fix a subchain $I_{k}$ and analyze system (3.1): it is a system of conservation laws in the variables $U=(\rho, \mu)$ :

$$
U_{t}+F(U)_{x}=0,
$$

with flux function given by $F(U)=(f(\rho, \mu),-\mu)$.

The eigenvalues and eigenvectors are given by:

$$
\begin{aligned}
& \lambda_{1}(\rho, \mu) \equiv-1, \quad r_{1}(\rho, \mu)= \begin{cases}\left(\begin{array}{c}
0 \\
1
\end{array}\right), & \text { if } \rho<\mu, \\
\left(\begin{array}{c}
-\frac{1-\varepsilon}{1+\varepsilon} \\
1
\end{array}\right), & \text { if } \rho>\mu,\end{cases} \\
& \lambda_{2}(\rho, \mu)= \begin{cases}1 & \text { if } \rho<\mu, \\
\varepsilon & \text { if } \rho>\mu,\end{cases}
\end{aligned}
$$

Hence the Hugoniot curves for the first family are vertical lines above the secant $\rho=\mu$ and lines with slope close to $-1 / 2$ below the same secant. The Hugoniot curves for the second family are just horizontal lines. Since we consider positive and bounded values for the variables, we fix the invariant region:

$$
\begin{aligned}
\mathcal{D}=\{( & (\rho, \mu): 0 \leq \rho \leq \rho_{\max }, 0 \leq \mu \leq \mu_{\max }, \\
& \left.0 \leq(1+\varepsilon) \rho+(1-\varepsilon) \mu \leq(1+\varepsilon) \rho_{\max }=2(1-\varepsilon) \mu_{\max }\right\} ;
\end{aligned}
$$

see Fig. 4 .

Observe that

$$
\rho_{\max }=\mu_{\max } \frac{2}{1+\varepsilon} .
$$

First we report some results proved in [9] for sequential supply chains.

Lemma 4.1. Given an initial datum $\left(\rho_{0}, \mu_{0}\right)$, the maximum value of the density of the curve of the second family passing through $\left(\rho_{0}, \mu_{0}\right)$ and belonging to the invariant region is given by

$$
\rho^{M}\left(\mu_{0}\right)=\rho_{\max }-\mu_{0} \frac{\rho_{\max }-\mu_{\max }}{\mu_{\max }} .
$$




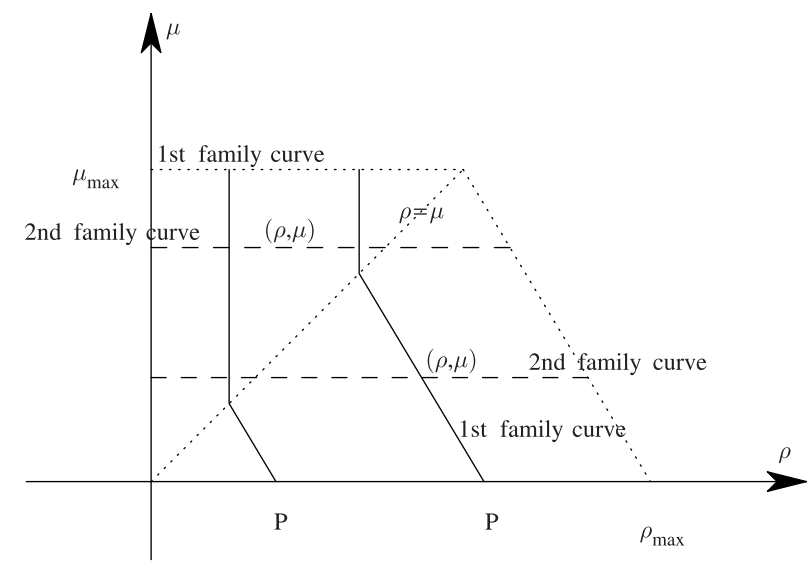

FIG. 4. First and second family curves.

Proposition 4.2. Given $\left(\rho_{0}, \mu_{0}\right)$, the minimal value of the flux at points of the curve of the first family passing through $\left(\rho_{0}, \mu_{0}\right)$ is given by:

$$
f^{\min }\left(\left(\rho_{0}, \mu_{0}\right)\right)= \begin{cases}\frac{2 \varepsilon}{1+\varepsilon} \rho_{0}, & \text { if } \rho_{0} \leq \mu_{0}, \\ \varepsilon \rho_{0}+\frac{\varepsilon(1-\varepsilon)}{1+\varepsilon} \mu_{0}, & \text { if } \rho_{0}>\mu_{0} .\end{cases}
$$

We consider a node $P$ with $n$ incoming subchains and $m$ outgoing ones and a Riemann initial datum $\left(\rho_{1,0}, \mu_{1,0}, \ldots, \rho_{n, 0}, \mu_{n, 0}, \rho_{n+1,0}, \mu_{n+1,0}, \ldots, \rho_{n+m, 0}, \mu_{n+m, 0}\right)$.

The following lemma holds:

LEMma 4.3. On the incoming subchains, only waves of the first family may be produced, while on the outgoing subchains only waves of the second family may be produced.

From Lemma 4.3 given the initial datum, for every Riemann Solver it follows that

$$
\begin{array}{ll}
\hat{\rho}_{i}=\varphi\left(\hat{\mu}_{i}\right), & i=1, \ldots, n, \\
\hat{\mu}_{j}=\mu_{j, 0}, & j=n+1, \ldots, n+m,
\end{array}
$$

where the function $\varphi(\cdot)$ describes the first family curve through $\left(\rho_{k, 0}, \mu_{k, 0}\right)$ as a function of $\hat{\mu}_{k}$. The expression of such a curve changes at a particular value $\bar{\mu}_{k}$, given by:

$$
\bar{\mu}_{k}= \begin{cases}\rho_{k, 0}, & \text { if } \rho_{k, 0} \leq \mu_{k, 0} \\ \frac{1+\varepsilon}{2} \rho_{k, 0}+\frac{1-\varepsilon}{2} \mu_{k, 0}, & \text { if } \rho_{k, 0}>\mu_{k, 0}\end{cases}
$$

We define two different Riemann Solvers at a junction that represents two different routing algorithms:

RA1. We assume that

(A) the flow from incoming subchains is distributed on outgoing subchains according to fixed coefficients;

(B) respecting (A) the processor chooses to process goods in order to maximize fluxes (i.e., the number of goods which are processed).

RA2. We assume that the number of goods through the junction is maximized both over incoming and outgoing subchains. 
For both routing algorithms we can maximize the flux of goods by considering one of the two additional rules, introduced in [9]:

SC2. The objects are processed in order to maximize the flux with the minimal value of the processing rate.

SC3. The objects are processed in order to maximize the flux. If a solution with only waves in the density $\rho$ exists, then such a solution is taken; otherwise the minimal $\mu$ wave is produced.

To define Riemann solvers according to rules RA1 and RA2 let us introduce the notation:

$$
f_{k}=f\left(\rho_{k}, \mu_{k}\right) .
$$

Define the maximum flux that can be obtained by a wave solution on each production subchain:

$$
f_{k}^{\max }= \begin{cases}\bar{\mu}_{k}, & k=1, \ldots, n, \\ \mu_{k, 0}+\varepsilon\left(\rho^{M}\left(\mu_{k, 0}\right)-\mu_{k, 0}\right), & k=n+1, \ldots, n+m .\end{cases}
$$

Since $\hat{f}_{i} \in\left[f_{i}^{\min }, f_{i}^{\max }=\bar{\mu}_{i}\right], i=1, \ldots, n$ and $\hat{f}_{j} \in\left[0, f_{j}^{\max }=\mu_{j, 0}+\varepsilon\left(\rho^{M}\left(\mu_{j, 0}\right)-\mu_{j, 0}\right)\right], j=$ $n+1, \ldots, n+m$ it follows that if

$$
\sum_{i=1}^{n} f_{i}^{\min }>\sum_{j=n+1}^{n+m} f_{j}^{\max }
$$

then the Riemann Problem does not admit a solution. Thus we get the following condition for the solvability of the supply network.

LEmma 4.4. A necessary and sufficient condition for the solvability of the Riemann Problems is that

$$
\sum_{i=1}^{n} f_{i}^{\text {min }} \leq \sum_{j=n+1}^{n+m} \mu_{j, 0}+\varepsilon\left(\rho^{M}\left(\mu_{j, 0}\right)-\mu_{j, 0}\right) .
$$

Lemma 4.5. A sufficient condition for the solvability of the Riemann Problems, independent of the initial data, is the following:

$$
\sum_{i=1}^{n} \rho_{i}^{\max } \leq \sum_{j=n+1}^{n+m} \mu_{j}^{\max } .
$$

Proof. Since $\hat{f}_{i} \in\left[f_{i}^{\min }, f_{i}^{\max }\right], i=1, \ldots, n$ and $\hat{f}_{j} \in\left[0, f_{j}^{\max }\right], j=n+1, \ldots, n+m$, the worst case to fulfill the condition of Lemma 4.4 happens when $f_{i}^{\min }$ assumes the greatest value and $f_{j}^{\max }$ the lowest one:

$$
\sum_{i=1}^{n} \varepsilon \rho_{i}^{\max } \leq \sum_{j=n+1}^{n+m} \varepsilon \mu_{j}^{\max } .
$$

In what follows, we first consider a single junction $P \in \mathcal{P}$ with $n-1$ incoming arcs and 1 outgoing arc (in short, a node of type $(n-1) \times 1)$ and then a junction with 1 incoming arc and $m-1$ outgoing ones (in short, a node of type $1 \times(m-1)$ ). 
4.1. One outgoing subchain. In this case the two algorithms RA1 and RA2 coincide since there is only one outgoing subchain. (See Fig. 5.)

We fix a node $P$ with $n-1$ incoming arcs and 1 outgoing one and a Riemann initial datum $\left(\rho_{0}, \mu_{0}\right)=\left(\rho_{1,0}, \mu_{1,0}, \ldots, \rho_{n-1,0}, \mu_{n-1,0}, \rho_{n, 0}, \mu_{n, 0}\right)$. Let us denote with $(\hat{\rho}, \hat{\mu})=$ $\left(\hat{\rho}_{1}, \hat{\mu}_{1}, \ldots, \hat{\rho}_{n-1}, \hat{\mu}_{n-1}, \hat{\rho}_{n}, \hat{\mu}_{n}\right)$ the solution of the Riemann Problem. In order to solve the dynamics we have to introduce the priority parameters $\left(q_{1}, q_{2}, \ldots, q_{n-1}\right)$ which determine a level of priority at the junction of incoming subchains.

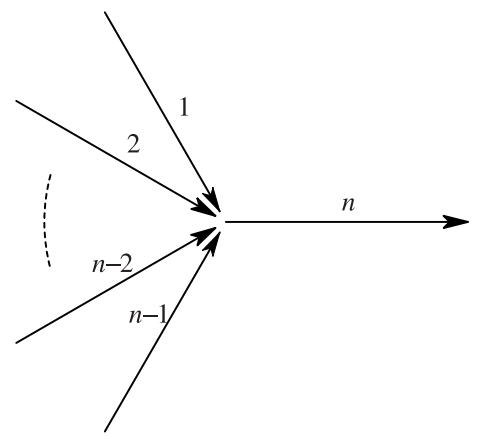

FIG. 5. One outgoing subchain.

Let us define

$$
\begin{aligned}
\Gamma_{\text {inc }} & =\sum_{i=1}^{n-1} f_{i}^{\max }, \\
\Gamma_{\text {out }} & =f_{n}^{\max },
\end{aligned}
$$

and $\Gamma=\min \left\{\Gamma_{\text {inc }}, \Gamma_{\text {out }}\right\}$.

We analyze for simplicity the case in which $n=3$; in this case, we need only one priority parameter $q \in] 0,1$. Think, for example, of a filling station for soda cans. The subchain 3 fills the cans, whereas subchains 1 and 2 produce plastic and aluminium cans, respectively.

First, we compute $\hat{f}_{i} i=1,2,3$ and then $\hat{\rho}_{i}$ and $\hat{\mu}_{i}, i=1,2,3$.

We have to distinguish two cases:

Case 1): $\Gamma=\Gamma_{\text {inc }}$.

Case 2): $\Gamma<\Gamma_{\text {inc }}$.

In the first case we set $\hat{f}_{i}=f_{i}^{\max }, i=1,2$. Let us analyze the second case in which we use the priority parameter $q$.

Not all objects can enter the junction, so let $C$ be the number of objects that can go through. Then $q C$ objects come from the first subchain and $(1-q) C$ objects from the second. Consider the space $\left(f_{1}, f_{2}\right)$ and define the following lines:

$$
\begin{aligned}
& r_{q}: f_{2}=\frac{1-q}{q} f_{1}, \\
& r_{\Gamma}: f_{1}+f_{2}=\Gamma .
\end{aligned}
$$


Define $P$ to be the point of intersection of the lines $r_{q}$ and $r_{\Gamma}$. Recall that the final fluxes should belong to the region (see Fig. 6):

$$
\Omega=\left\{\left(f_{1}, f_{2}\right): 0 \leq f_{i} \leq f_{i}^{\max }, i=1,2\right\} .
$$

We distinguish two cases:

a) $P$ belongs to $\Omega$,

b) $P$ is outside $\Omega$.

In the first case we set $\left(\hat{f}_{1}, \hat{f}_{2}\right)=P$, while in the second case we set $\left(\hat{f}_{1}, \hat{f}_{2}\right)=Q$, with $Q=\operatorname{proj}_{\Omega \cap r_{\Gamma}}(P)$ where proj is the usual projection on a convex set; see Fig. 6 .

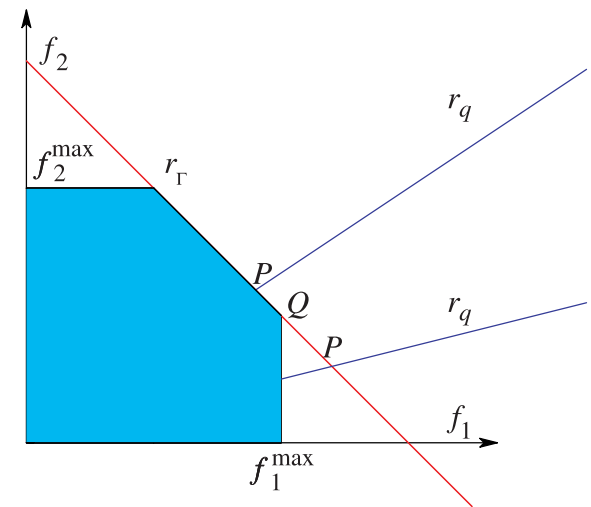

FIG. 6. $P$ belongs to $\Omega$ and $P$ is outside $\Omega$.

Notice that $\hat{f}_{3}=\Gamma$.

REMARK 4.6. The reasoning can be repeated also in the case of $n-1$ incoming subchains. In $\mathbb{R}^{n-1}$ the line $r_{q}$ is given by $r_{q}=t v_{q}, t \in \mathbb{R}$, with $v_{q} \in \Delta_{n-2}$, where

$$
\Delta_{n-2}=\left\{\left(f_{1}, \ldots, f_{n-1}\right): f_{i} \geq 0, i=1, \ldots, n-1, \sum_{i=1}^{n-1} f_{i}=1\right\}
$$

is the $(n-2)$-dimensional simplex and

$$
H_{\Gamma}=\left\{\left(f_{1}, \ldots, f_{n-1}\right): \sum_{i=1}^{n-1} f_{i}=\Gamma\right\}
$$

is a hyperplane. Since $v_{q} \in \Delta_{n-2}$, there exists a unique point $P=r_{q} \cap H_{\Gamma}$. If $P \in \Omega$, then we set $\left(\hat{f}_{1}, \ldots, \hat{f}_{n-1}\right)=P$. If $P \notin \Omega$, then we set $\left(\hat{f}_{1}, \ldots, \hat{f}_{n-1}\right)=Q=\operatorname{proj}_{\Omega \cap H_{\Gamma}}(P)$, the projection over the subset $\Omega \cap H_{\Gamma}$. Observe that the projection is unique since $\Omega \cap H_{\Gamma}$ is a closed convex subset of $H_{\Gamma}$.

Let us compute $\hat{\rho}_{k}$ and $\hat{\mu}_{k}, k=1,2,3$.

On the incoming subchains we have to distinguish two subcases: 
Case 2.1): $\hat{f}_{i}=f_{i}^{\max }$. We set according to rules SC2 and SC3,

$$
\begin{array}{ll} 
& \hat{\rho}_{i}=\bar{\mu}_{i}, \quad i=1,2, \\
\mathrm{SC} 2: & \hat{\mu}_{i}=\bar{\mu}_{i}, \\
\mathrm{SC} 3: & \hat{\rho}_{i}=\bar{\mu}_{i}, \\
& \hat{\mu}_{i}=\max \left\{\bar{\mu}_{i}, \mu_{i, 0}\right\}, \quad i=1,2 .
\end{array}
$$

Case 2.2): $\hat{f}_{i}<f_{i}^{\max }$. In this case there exists a unique $\hat{\mu}_{i}$ such that $\hat{\mu}_{i}+$ $\varepsilon\left(\varphi\left(\hat{\mu}_{i}\right)-\hat{\mu}_{i}\right)=\hat{f}_{i}$. According to (4.2), we set $\hat{\rho}_{i}=\varphi\left(\hat{\mu}_{i}\right), i=1,2$.

Observe that in case 2.1), $\hat{\rho}_{i}=\varphi\left(\hat{\mu}_{i}\right)=\bar{\mu}_{i}, i=1,2$.

On the outgoing subchain we have

$$
\hat{\mu}_{3}=\mu_{3,0},
$$

while $\hat{\rho}_{3}$ is the unique value such that $f_{\varepsilon}\left(\mu_{3,0}, \hat{\rho}_{3}\right)=\hat{f}_{3}$.

4.2. One incoming subchain. We fix a node $P$ with 1 incoming arc and $m-1$ outgoing ones and a Riemann initial datum $\left(\rho_{0}, \mu_{0}\right)=\left(\rho_{1,0}, \mu_{1,0}, \rho_{2,0}, \mu_{2,0}, \ldots, \rho_{m, 0}, \mu_{m, 0}\right)$. (See Fig.77) Let us denote with $(\hat{\rho}, \hat{\mu})=\left(\hat{\rho}_{1}, \hat{\mu}_{1}, \hat{\rho}_{2}, \hat{\mu}_{2}, \ldots, \hat{\rho}_{m}, \hat{\mu}_{m}\right)$ the solution of the Riemann Problem. Since we have more than one outgoing arc, we need to define the distribution of goods from the incoming arc.

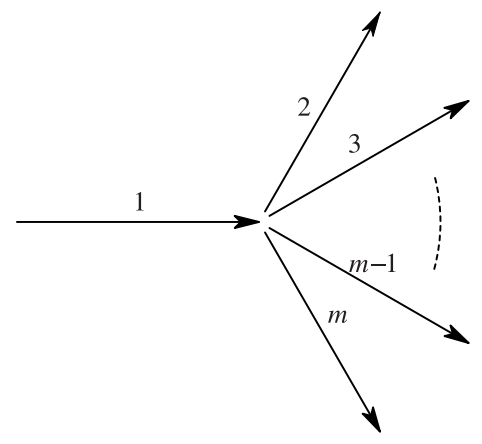

FIG. 7. One incoming subchain.

Introduce the flux distribution parameters $\alpha_{j}, j=2, \ldots, m$, where

$$
0<\alpha_{j}<1, \sum_{j=2}^{m} \alpha_{j}=1 .
$$

The coefficient $\alpha_{j}$ denotes the percentage of objects addressed from the arc 1 to the subchain $j$. The flux on the $\operatorname{arc} j$ is thus given by

$$
f_{j}=\alpha_{j} f_{1}, j=2, \ldots, m,
$$

where $f_{1}$ is the incoming flux on the arc 1 . 
Let us define

$$
\begin{aligned}
& \Gamma_{\text {inc }}=f_{1}^{\max }, \\
& \Gamma_{\text {out }}=\sum_{j=2}^{m} f_{j}^{\max },
\end{aligned}
$$

and $\Gamma=\min \left\{\Gamma_{\text {inc }}, \Gamma_{\text {out }}\right\}$.

We have to determine $\hat{\mu}_{k}$ and $\hat{\rho}_{k}, k=1, \ldots, m$ for both algorithms RA1 and RA2.

4.2.1. Riemann Solver according to RA1. Analyze the general case with $m$ subchains. Consider, for example, the filling station for wine bottles of Section 2. The subchains $I_{3}$ and $I_{4}$ fill bottles with white and red wines, respectively, according to the bottle shapes. The dynamics at node 2 is solved using the algorithm we are going to describe. Since $\hat{f}_{j} \leq f_{j}^{\max }$ it follows that

$$
\hat{f}_{1} \leq \frac{f_{j}^{\max }}{\alpha_{j}}, \quad j=2, \ldots, m
$$

We set

$$
\begin{aligned}
& \hat{f}_{1}=\min \left\{f_{1}^{\max }, \frac{f_{j}^{\max }}{\alpha_{j}}\right\}, \quad j=2, \ldots, m . \\
& \hat{f}_{j}=\alpha_{j} \hat{f}_{1},
\end{aligned}
$$

On the incoming subchain we have to distinguish two subcases:

Case 1): $\hat{f}_{1}=f_{1}^{\max }$. According to rules SC2 and SC3, respectively, we set

$$
\begin{array}{ll}
\mathrm{SC} 2: & \hat{\rho}_{1}=\bar{\mu}_{1}, \\
& \hat{\mu}_{1}=\bar{\mu}_{1}, \\
\mathrm{SC} 3: & \hat{\rho}_{1}=\bar{\mu}_{1}, \\
& \hat{\mu}_{1}=\max \left\{\bar{\mu}_{1}, \mu_{1,0}\right\} .
\end{array}
$$

Case 2): $\hat{f}_{1}<f_{1}^{\max }$. In this case there exists a unique $\hat{\mu}_{1}$ such that $\hat{\mu}_{1}+$ $\varepsilon\left(\varphi\left(\hat{\mu}_{1}\right)-\hat{\mu}_{1}\right)=\hat{f}_{1}$. According to (4.2), we set $\hat{\rho}_{1}=\varphi\left(\hat{\mu}_{1}\right)$.

On the outgoing subchain we have

$$
\hat{\mu}_{j}=\mu_{j, 0}, \quad j=2,3,
$$

while $\hat{\rho}_{i}$ is the unique value such that $f_{\varepsilon}\left(\mu_{j, 0}, \hat{\rho}_{j}\right)=\hat{f}_{j}, j=2,3$.

4.2.2. Riemann Solver according to RA2. Let us analyze for simplicity the case in which $m=3$; in this case we need only one distribution parameter $\alpha \in] 0,1[$. Think, for example, of the supply network of beach production described in Section 2 . The dynamics at the node is solved according to the algorithm RA2. Compute $\hat{f}_{k}, k=1,2,3$.

We have to distinguish two cases:

Case 1): $\Gamma=\Gamma_{\text {out }}$.

Case 2): $\Gamma<\Gamma_{\text {out }}$.

In the first case we set $\hat{f}_{j}=f_{j}^{\max }, j=2,3$. Let us analyze the second case in which we use the priority parameter $\alpha$.

Not all objects can enter the junction, so let $C$ be the number of objects that can go through. Then $\alpha C$ objects come from the first subchain and $(1-\alpha) C$ objects from the 
second. Consider the space $\left(f_{2}, f_{3}\right)$ and define the following lines:

$$
\begin{aligned}
& r_{\alpha}: f_{3}=\frac{1-\alpha}{\alpha} f_{2}, \\
& r_{\Gamma}: f_{2}+f_{3}=\Gamma .
\end{aligned}
$$

Define $P$ to be the point of intersection of the lines $r_{\alpha}$ and $r_{\Gamma}$. Recall that the final fluxes should belong to the region:

$$
\Omega=\left\{\left(f_{2}, f_{3}\right): 0 \leq f_{j} \leq f_{j}^{\max }, j=2,3\right\} .
$$

We distinguish two cases:

a) $P$ belongs to $\Omega$,

b) $P$ is outside $\Omega$.

In the first case we set $\left(\hat{f}_{2}, \hat{f}_{3}\right)=P$, while in the second case we set $\left(\hat{f}_{2}, \hat{f}_{3}\right)=Q$, with $Q=\operatorname{proj}_{\Omega \cap r_{\Gamma}}(P)$ where proj is the usual projection on a convex set. Observe that $\hat{f}_{1}=\Gamma$.

Again, we can extend the reasoning to the case of $m-1$ outgoing subchains as for the incoming subchains defining the hyperplane

$$
H_{\Gamma}=\left\{\left(f_{2}, \ldots, f_{m}\right): \sum_{j=2}^{m} f_{j}=\Gamma\right\}
$$

and choosing a vector $v_{\alpha} \in \Delta_{m-2}$. Moreover, we compute $\hat{\rho}_{k}$ and $\hat{\mu}_{k}$ in the same way described for the Riemann Solver RA1.

REMARK 4.7. An alternative way of choosing the vector $v_{\alpha}$ is the following. We assume that a traffic distribution matrix $A$ is assigned. Then we compute $\hat{f}_{1}$, and choose $v_{\alpha} \in \Delta_{m-2}$ by

$$
v_{\alpha}=\Delta_{m-2} \cap\left\{t A\left(\hat{f}_{1}\right): t \in \mathbb{R}\right\} .
$$

REMARK 4.8. The classical Kruzkov entropy inequalities at nodes ([5]) read

$$
\sum_{\text {inc }} \operatorname{sgn}(\rho-k)(f(\rho)-f(k)) \geq \sum_{\text {out }} \operatorname{sgn}(\rho-k)(f(\rho)-f(k)),
$$

where the sums are respectively over incoming and outgoing subchains and $k$ is arbitrary. The fluxes are always monotone with respect to $\rho$, while the precise values taken by fluxes and densities on the subchains may be different. Thus we cannot expect the inequality to hold in general.

4.3. Waves production. Let us now discuss the waves production on an incoming subchain and on an outgoing one with initial data $\left(\rho_{i, 0}, \mu_{i, 0}\right)$ and $\left(\rho_{j, 0}, \mu_{j, 0}\right)$, respectively. Since the load dynamics is described by a conservation law in $\rho$ and an evolution equation in $\mu$, we have $\rho$-waves and $\mu$-waves of two types: shocks waves which are discontinuities in $\rho$ and/or $\mu$ travelling at a constant speed, and contact discontinuities, which separate two constant states with the same speed but different values. In particular, on an incoming subchain only waves of the first family can be produced. They are contact discontinuities in $\rho$ and $\mu$ with speed $\lambda=-1$ connecting the states $\rho_{i, 0}$ and $\hat{\rho}_{i}$ and $\mu_{i, 0}$ and $\hat{\mu}_{i}$. 
On the outgoing subchain only $\rho$-waves of the second family can be produced. Two cases must be considered:

Case a): $\rho_{j, 0} \leq \mu_{j, 0}$,

Case b): $\rho_{j, 0}>\mu_{j, 0}$.

In case a) we have to distinguish two subcases:

Case a1): If $\hat{\rho}_{j} \in\left[0, \mu_{j, 0}\right]$, then the solution of the RP consists of a contact discontinuity connecting $\hat{\rho}_{j}$ and $\rho_{j, 0}$ with speed 1 (for $t=1$ ).

Case a2): If $\left.\left.\hat{\rho}_{j} \in\right] \mu_{j, 0}, \mu_{j}^{\max }\right]$, then the solution of the RP consists of two shocks: one connecting $\hat{\rho}_{j}$ and $\mu_{j, 0}$ with speed $\varepsilon$ (for $t=1$ ) followed by another shock connecting $\mu_{j, 0}$ and $\rho_{j, 0}$ travelling with speed 1 (for $t=1$ ); see Fig. 8 .

In case b) we have to consider two subcases:

Case b1): If $\hat{\rho}_{j} \in\left[0, \mu_{j, 0}\right]$, then the solution of the $\mathrm{RP}$ consists of a shock wave connecting the states $\hat{\rho}_{j}$ and $\rho_{j, 0}$ with speed (for $t=1$ ) equal to the slope $\lambda$ of the line connecting the two states,

$$
\lambda=\frac{\mu_{j, 0}+\varepsilon\left(\rho_{j, 0}-\mu_{j, 0}\right)-\hat{\rho}_{j}}{\rho_{j, 0}-\hat{\rho}_{j}} .
$$

Case b2): If $\left.\left.\hat{\rho}_{j} \in\right] \mu_{j, 0}, \mu_{j}^{\max }\right]$, then the solution of the RP consists of a contact discontinuity connecting $\hat{\rho}_{j}$ and $\rho_{j, 0}$ with speed $\varepsilon$ (for $t=1$ ).
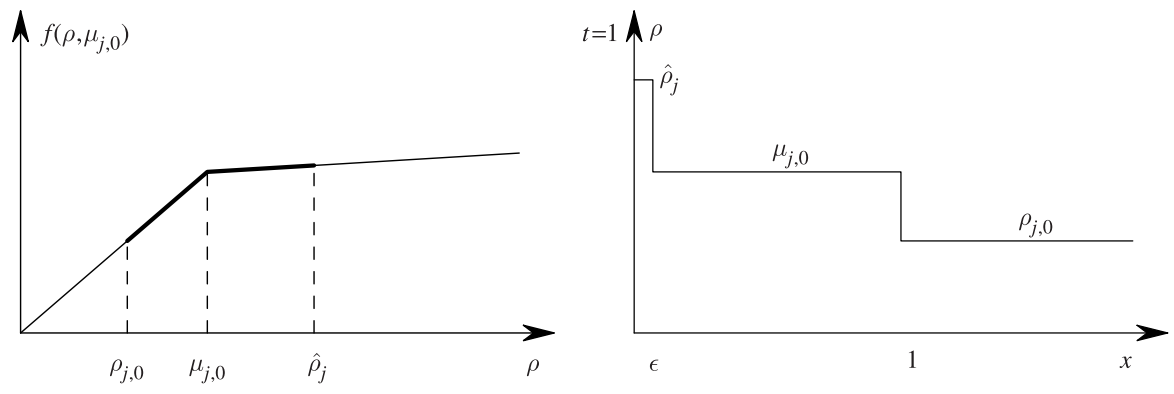

FIG. 8. Wave production on an outgoing subchain: Case a2).

In what follows we report the densities and production rates at the instant $t=0$ and after some time (at $t=1$ ) for different initial data using different routing algorithms. Since a constant state is an equilibrium for the single line model, a modification of the state may only appear initially at the junction. In Table 1 and in Figs. 9 10 we report the Riemann Solver for a node of type $1 \times 2$ and assume $\varepsilon=0.2, \mu_{i}^{\max }=1, i=$ $1,2,3, \alpha=0.8,\left(\rho_{1,0}, \rho_{2,0}, \rho_{3,0}\right)=(0.7,0.1,0),\left(\mu_{1,0}, \mu_{2,0}, \mu_{3,0}\right)=(1,0.2,1)$. Observe that the algorithm RA2 redirects the goods; in fact taking into account the initial loads of the outgoing subchains, the number of goods processed by the subchain with density $\rho_{3,0}=0$ increases.

In Table 2 and in Figs. 1142 we report numerical results for a node of type $2 \times$ 1 , and assume $\varepsilon=0.2, \mu_{i}^{\max }=1, i=1,2,3, q=0.6,\left(\rho_{1,0}, \rho_{2,0}, \rho_{3,0}\right)=(0.3,0.7,0.8)$, $\left(\mu_{1,0}, \mu_{2,0}, \mu_{3,0}\right)=(0.8,0.7,0.4)$. 
TABLE 1. A node of type $1 \times 2$

\begin{tabular}{||c|c|c|c|c||}
\hline \hline \multirow{2}{*}{} & \multicolumn{2}{|c|}{ RA1 } & \multicolumn{2}{c||}{ RA2 } \\
\cline { 2 - 5 } & SC2 & SC3 & SC2 & SC3 \\
\hline$\hat{f}_{i}$ & $(0.58,0.47,0.12)$ & $(0.58,0.47,0.12)$ & $(0.7,0.47,0.23)$ & $(0.7,0.47,0.23)$ \\
\hline$\hat{\rho}_{i}$ & $(0.82,1.53,0.12)$ & $(0.82,1.53,0.12)$ & $(0.7,1.53,0.23)$ & $(0.7,1.53,0.23)$ \\
\hline$\hat{\mu}_{i}$ & $(0.52,0.2,1)$ & $(0.52,0.2,1)$ & $(0.7,0.2,1)$ & $(1,0.2,1)$ \\
\hline \hline
\end{tabular}

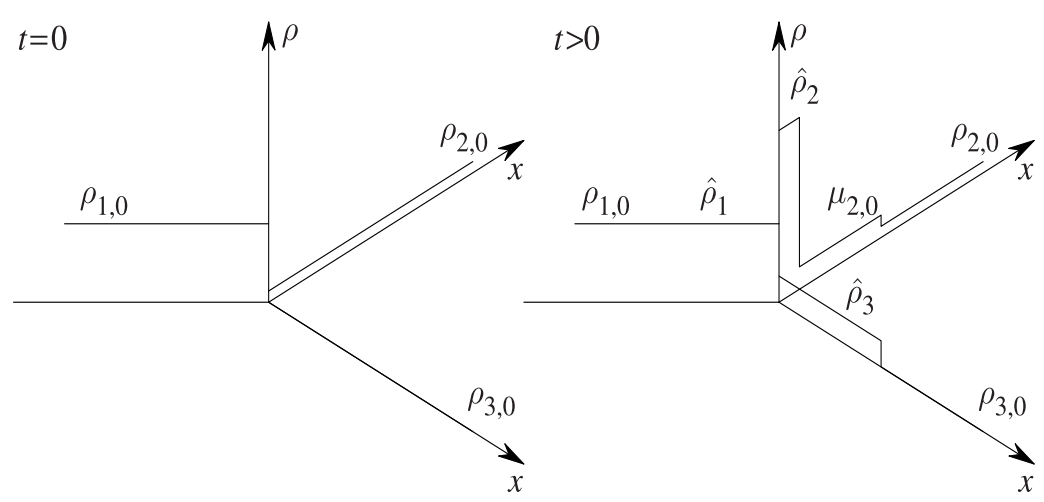

FIG. 9. An RP for the RA2-SC3 algorithm: The initial density and the density after some time.

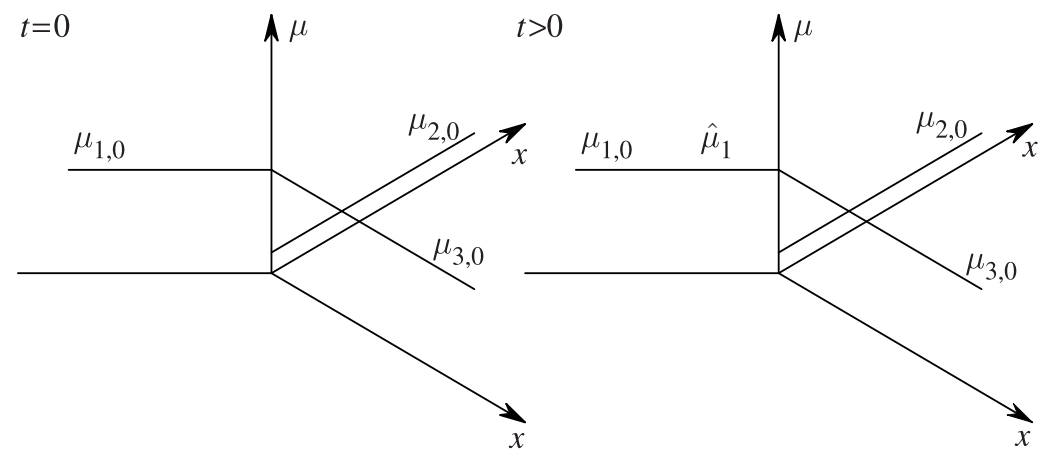

FIG. 10. An RP for the RA2-SC3 algorithm: The initial production rate and the production rate after some time.

TABle 2. A node of type $2 \times 1$

\begin{tabular}{||c|c|c||}
\hline \hline \multirow{2}{*}{} & \multicolumn{2}{|c||}{ RA1=RA2 } \\
\cline { 2 - 3 } & SC2 & SC3 \\
\hline$\hat{f}_{i}$ & $(0.3,0.3,0.6)$ & $(0.3,0.3,0.6)$ \\
\hline$\hat{\rho}_{i}$ & $(0.3,1.1,1.4)$ & $(0.3,1.1,1.4)$ \\
\hline$\hat{\mu}_{i}$ & $(0.3,0.1,0.4)$ & $(0.8,0.1,0.4)$ \\
\hline \hline
\end{tabular}




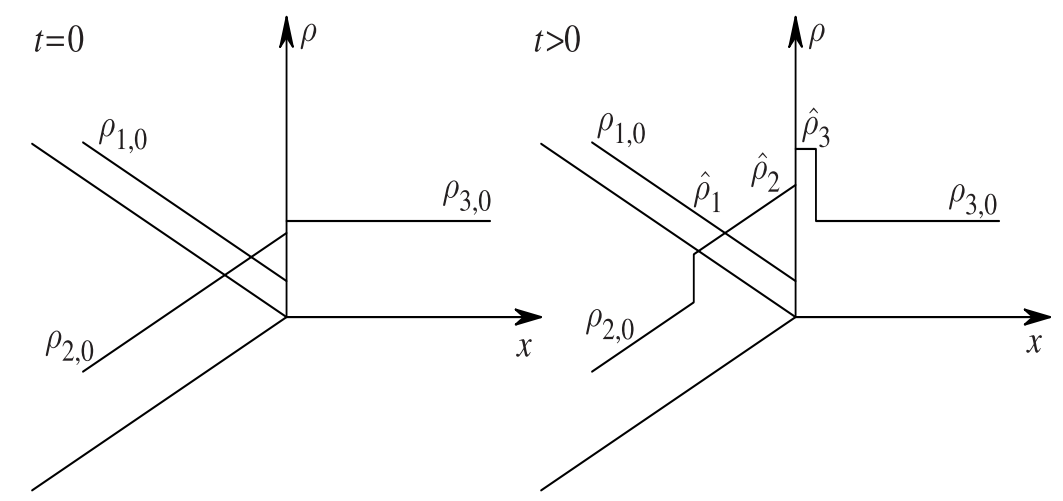

FIG. 11. An RP for the SC2 algorithm: The initial density and the density after some time.

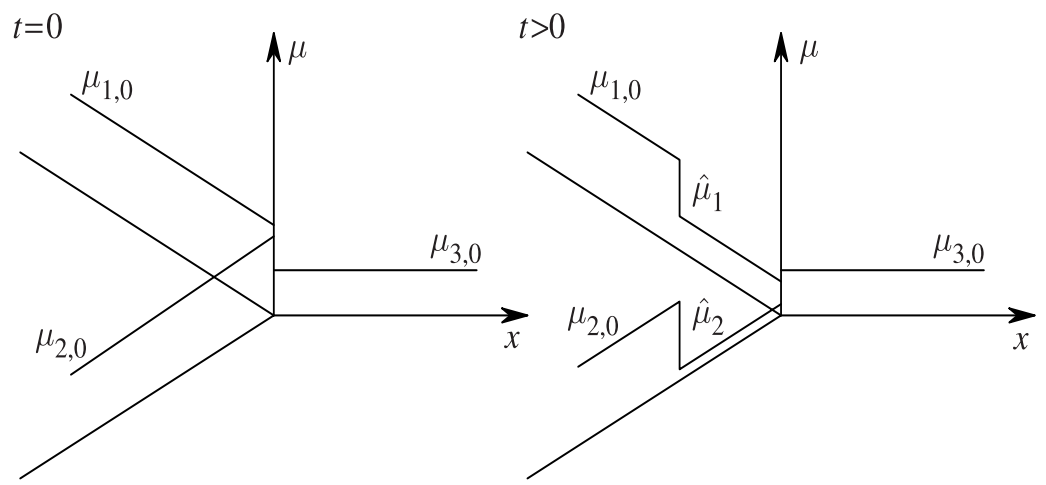

FIG. 12. An RP for the SC2 algorithm: The initial production rate and the production rate after some time.

5. Equilibrium analysis. In this section we discuss the equilibria at nodes. We fix a node $P$ and a Riemann initial datum $\left(\rho_{0}, \mu_{0}\right)$.

Definition 5.1. Define $(\hat{\rho}, \hat{\mu})=R S\left(\left(\rho_{0}, \mu_{0}\right)\right)$. The datum $\left(\rho_{0}, \mu_{0}\right)$ is an equilibrium if

$$
(\hat{\rho}, \hat{\mu})=R S\left(\left(\rho_{0}, \mu_{0}\right)\right)=\left(\rho_{0}, \mu_{0}\right) .
$$

We consider generic equilibria for the Riemann Problem at a junction. Let us distinguish two types of nodes, $(n-1) \times 1$ and $1 \times(m-1)$, and equilibria with active and not active constraints for the maximization problem.

5.1. A node with one outgoing subchain. If the $n$-th subchain is an active constraint, then we have

$$
\rho_{n}=\rho^{M}\left(\mu_{n}\right) ;
$$

otherwise, if it is not an active constraint, we have

$$
\rho_{n}<\rho^{M}\left(\mu_{n}\right) .
$$


For the incoming subchains $I_{i}, i=1, \ldots, n-1$, we have the following. If the $i$-th subchain is an active constraint, then

$$
\begin{array}{ll}
\mathrm{SC} 2: & \mu_{i}=\rho_{i}, \quad i=1, \ldots, n-1 . \\
\mathrm{SC} 3: & \mu_{i} \geq \rho_{i},
\end{array}
$$

Otherwise

$$
\rho_{i} \geq \mu_{i}
$$

The equilibria are reported in Figures 13 and 14. In the latter the equilibria for the algorithm SC2 are depicted in bold, and those for the algorithm SC3 in bold and grey.

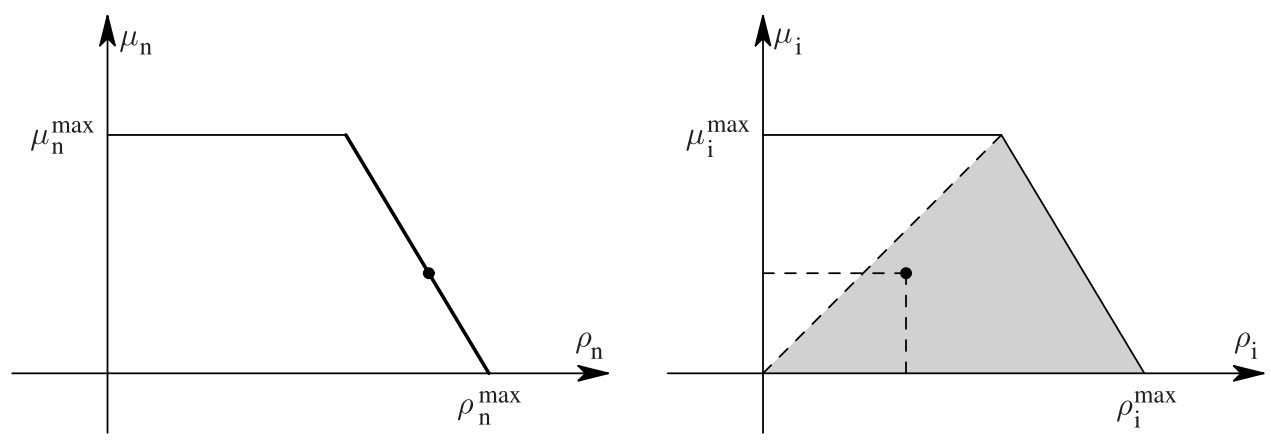

FIG. 13. The outgoing subchain is an active constraint and the incoming ones are not active constraints.
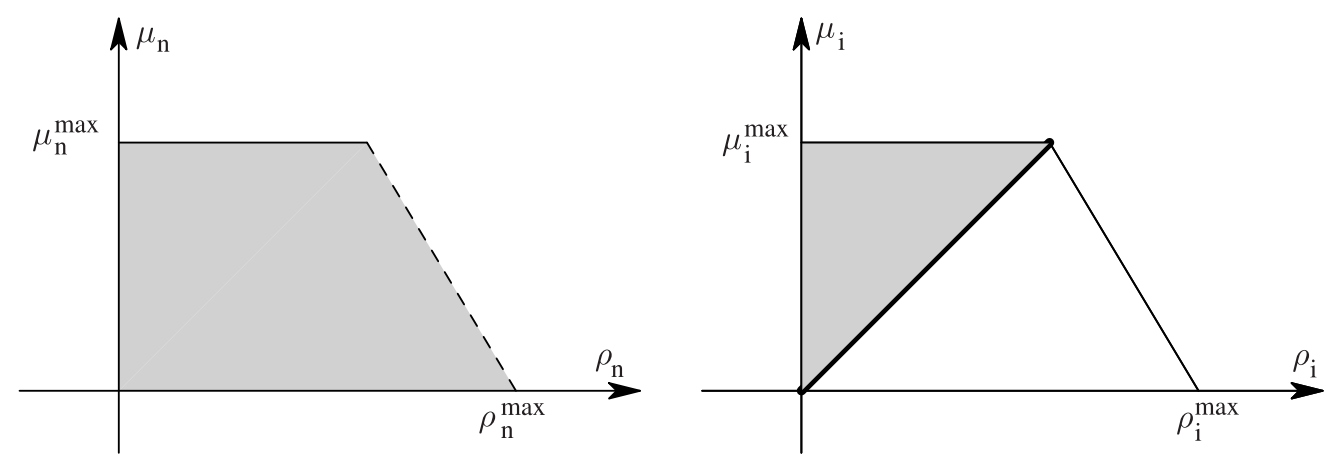

FIG. 14. The incoming subchains are active constraints and the outgoing one is not an active constraint.

The first type of equilibria (Figure 13) represents the situation in which the outgoing subchain exhibits the maximal production effort, while the incoming subchains adjust accordingly their production flows. In practice we expect this situation to show up frequently.

The second type of equilibria (Figure 14) represents the situation in which the incoming subchains have a low level of part densities and, consequently, the outgoing subchain is not used at maximal level. In other words, the whole plant is not used in an appropriate way and a rebuilding is in order. Either the incoming subchains should be powered or 
the outgoing ones should be restricted. The first solution would improve the production rate, while the second would lower the production costs.

5.2. A node with one incoming subchain. The equilibria for the two algorithms RA1 and RA2 coincide. In particular, if the incoming subchain is an active constraint, then

$$
\begin{array}{ll}
\text { SC2 : } & \rho_{1}=\mu_{1}, \\
\text { SC3 : } & \rho_{1} \leq \mu_{1} ;
\end{array}
$$

otherwise $\rho_{1} \geq \mu_{1}$. For the outgoing subchains $I_{j}, j=2, \ldots, m$, the following holds. If $I_{j}$ is an active constraint, then $\rho_{j}=\rho^{M}\left(\mu_{j}\right)$, for both SC2 and SC3 algorithms. Otherwise $\rho_{j}<\rho^{M}\left(\mu_{j}\right)$.

For both algorithms RA1 and RA2, the case of an incoming subchain as active constraints should happen only with $\rho_{1}=\mu_{1}$, in such a way that the goods fill up appropriately the subchain. Otherwise the incoming subchain should be powered.

The situation for outgoing subchains as active constraints is different. In fact, the latter represents a projecting error for the algorithm RA1, while it may well happen for RA2.

5.3. Bullwhip effect. The Bullwhip effect is a well-known oscillation phenomenon in supply chain theory; see [8]. Since the effect consists in oscillations moving backwards, we restrict ourselves to the most interesting case of nodes with $n-1$ incoming subchains and one outgoing subchain.

To study the Bullwhip effect, we compute the oscillations on incoming subchains produced by the interaction with the node of a wave from the outgoing one. Since the wave must have negative speed, it is a first family wave. To fix notation, let $\left(\rho^{-}, \mu^{-}\right)$be an equilibrium configuration at the node and $\left(\left(\rho_{n}^{-}, \mu_{n}^{-}\right),\left(\tilde{\rho}_{n}, \tilde{\mu}_{n}\right)\right)$ the wave coming to the node. In general, we denote with - and + the values before and after the interaction, while by $\Delta$ we indicate the jump in the values from the left to the right along waves travelling on subchains.

The effect of the interaction of the wave is the production of $n-1$ waves on the incoming subchains.

The oscillation amplitude in the production rate before the interaction is given by

$$
\Delta \mu^{-}=\tilde{\mu}_{n}-\mu_{n}^{-} .
$$

The maximum flux on the outgoing subchain as a function of $\mu$ is the following:

$$
f_{n}^{\max }(\mu)=\mu \frac{1-\varepsilon}{1+\varepsilon}+\varepsilon \rho_{n}^{\max }
$$

thus it is an increasing function. It follows that the oscillation of the flux after the interaction is

$$
\Delta f^{+}=\frac{1-\varepsilon}{1+\varepsilon} \Delta \mu^{-} .
$$

Assume first that the incoming subchains are not active constraints. Then for both algorithms SC2 and SC3, we have $\rho_{i}^{-} \geq \mu_{i}^{-}, i=1, \ldots, n-1$. Then the first family curve passing through $\left(\rho_{i}^{-}, \mu_{i}^{-}\right)$, belonging to the region $\rho \geq \mu$, is given by

$$
\rho=\rho_{i}^{-}+\left(\mu-\mu_{i}^{-}\right)\left(-\frac{1-\varepsilon}{1+\varepsilon}\right),
$$


from which, for small oscillations we obtain

$$
\Delta \rho^{+}=-\frac{1-\varepsilon}{1+\varepsilon} \Delta \mu^{+} .
$$

If the oscillation is not small the same relation holds with an inequality sign. Observe that

from which

$$
\Delta f^{+}=\Delta \mu^{+}(1-\varepsilon)+\varepsilon \Delta \rho^{+}=\frac{1-\varepsilon}{1+\varepsilon} \Delta \mu^{+},
$$

and then

$$
\Delta \mu^{+}=\frac{1+\varepsilon}{1-\varepsilon} \Delta f^{+}
$$

$$
\Delta \mu^{+}=\Delta \mu^{-} .
$$

Assume now that the incoming subchains are active constraints. This means that $\mu_{i}^{-}=\rho_{i}^{-}$for the SC2 algorithm and $\mu_{i}^{-} \geq \rho_{i}^{-}$for the SC3 algorithm. Along the curve of the first family belonging to the region $\rho \leq \mu$ we have $\Delta f=0$; i.e., a dumping effect is possible. On the contrary, in the region $\rho \geq \mu$ we have

$$
\Delta f=\frac{1-\varepsilon}{1+\varepsilon} \Delta \mu
$$

Consider first the case of the $\mathrm{SC} 2$ algorithm. In case the first family wave from the outgoing road increases the flux, then it is reflected as a second family wave. In the opposite case, we get the same estimates as above.

Consider now the case of the SC3 algorithm. In case the first family wave from the outgoing road increases the flux, then it is again reflected as a second family wave. In the opposite case, we get:

$$
\Delta \mu^{+}=\Delta \mu^{-}+\left(\mu_{i}^{-}-\rho_{i}^{-}\right)
$$

with an increase in the production rate oscillation.

Concluding we get the following:

Proposition 5.2. The algorithm SC3 may produce the Bullwhip effect. On the contrary, the algorithm SC2 conserves oscillations or produces a dumping effect, thus not permitting the Bullwhip effect.

\section{REFERENCES}

[1] D. Armbruster, C. de Beer, M. Freitag, T. Jagalski and C. Ringhofer: Autonomous Control of Production Networks using a Pheromone Approach. Physica A: Statistical Mechanics and its applications, Vol. 363, Issue 1, pp. 104-114, 2006.

[2] D. Armbruster, P. Degond and C. Ringhofer: A model for the dynamics of large queuing networks and supply chains. SIAM Journal on Applied Mathematics, Vol. 66, Issue 3, pp. 896-920, 2006. MR.2216725 (2006k:90015)

[3] D. Armbruster, P. Degond and C. Ringhofer: Kinetic and fluid models for supply chains supporting policy attributes. Bull. Inst. Math. Acad. Sin. (N.S.), Vol. 2(2), pp. 433-460, 2007. MR2325723 (2008e:90031)

[4] D. Armbruster, D. Marthaler and C. Ringhofer: Kinetic and fluid model hierarchies for supply chains. SIAM J. on Multiscale Modeling, Vol. 2(1), pp. 43-61, 2004. MR2044956 (2004m:90061)

[5] A. Bressan: Hyperbolic Systems of Conservation Laws. The One-dimensional Cauchy Problem. Oxford Univ. Press, 2000. MR.1816648 (2002d:35002)

[6] G. Bretti, C. D'Apice, R. Manzo, and B. Piccoli: A continuum-discrete model for supply chains dynamics. Networks and Heterogeneous Media, Vol. 2, No. 4, pp. 661-694, 2007. MR 2357763 
[7] C. Dafermos: Hyperbolic Conservation Laws in Continuum Physics. Springer-Verlag, 1999. MR 2169977 (2006d:35159)

[8] C. F. Daganzo: A Theory of Supply Chains. Springer Verlag, New York, Berlin, Heidelberg, 2003. MR1968682(2004b:90003)

[9] C. D'Apice and R. Manzo: A fluid dynamic model for supply chains. Networks and Heterogeneous Media, Vol. 1, No. 3, pp. 379-398, 2006. MR2247783 (2007f:35184)

[10] C. D'Apice, R. Manzo, and B. Piccoli: Packets Flow on Telecommunication Networks. SIAM J. on Math. Anal., Vol. 38 (3), pp. 717-740, 2006. MR2262939 (2007h:35221)

[11] D. Helbing, S. Lämmer, T. Seidel, P. Seba, and T. Platkowski: Physics, stability and dynamics of supply networks. Physical Review E 70, 2004, 066116.

[12] D. Helbing and S. Lämmer: Supply and production networks: From the bullwhip effect to business cycles, in: D. Armbruster, A. S. Mikhailov, and K. Kaneko (eds.) Networks of Interacting Machines: Production Organization in Complex Industrial Systems and Biological Cells, World Scientific, Singapore, pp. 33-66, 2005.

[13] S. Gottlich, M. Herty and A. Klar: Network models for supply chains. Comm. Math. Sci., Vol. 3(4), pp. 545-559, 2005. MR2188683 (2006f:90004)

[14] S. Gottlich, M. Herty and A. Klar: Modelling and Optimization of Supply Chains on Complex Networks. Comm. Math. Sci., Vol. 4 (2), pp. 315-330, 2006. MR2219354 (2006m:90073)

[15] M. Herty, A. Klar, and B. Piccoli: Existence of solutions for supply chain models based on partial differential equations. SIAM J. Math. Anal., Vol. 39, No. 1, pp. 160-173, 2007. MR2318380

[16] M. J. Lighthill and G. B. Whitham: On kinetic waves. II. Theory of Traffic Flows on Long Crowded Roads. Proc. Roy. Soc. London Ser. A, 229, pp. 317-345, 1955. MR0072606 (17:310a)

[17] E. Mosekilde and E.R. Larsen: System Dynamics Rev., Vol. 4, 1/2, pp. 131-147, 1988.

[18] T. Nagatani and D. Helbing: Stability analysis and stabilization strategies for linear supply chains, Physica A: Statistical and Theoretical Physics, Vol. 335, Issues 3-4, pp. 644-660, 2004. MR2044162

[19] P. I. Richards: Shock Waves on the Highway. Oper. Res., 4, pp. 42-51, 1956. MR0075522 (17:761b)

[20] J.D. Sterman: Business Dynamics, McGraw-Hill, Boston, 2000.

[21] http://www.sancarlo.it/it/publishing2.asp?ArticleId=5. 